\title{
Android-based Application Utilizing Image Processing with Artificial Neural Network for Detecting Ringworm and Yeast Infections for Dogs Using Neuroph Framework
}

\author{
Reand Michael M. Mellores ${ }^{1}$, Jessa Carrisse D. Salvoza ${ }^{2}$, Shantal O. Flores ${ }^{3}$, Jan Carlo T. Arroyo ${ }^{4}$, \\ Allemar Jhone P. Delima ${ }^{5}$ \\ ${ }^{1-5}$ College of Computing Education, University of Mindanao, Davao City, Davao del Sur, Philippines \\ ${ }^{5}$ College of Engineering, Technology and Management, Cebu Technological University-Barili Campus, \\ Cebu, Philippines \\ reand_mellores@umindanao.edu.ph ${ }^{1}$, jessa_salvoza@umindanao.edu.ph ${ }^{2}$, shantal_flores@umindanao.edu.ph ${ }^{3}$, \\ jancarlo_arroyo@umindanao.edu.ph ${ }^{4}$, allemarjpdjca@yahoo.com ${ }^{5}$
}

\begin{abstract}
This study offers dog owners a system deeloped using Android that can determine a dog's skin infections such as yeast and ringworm with the use of an image processing algorithm. The system can run on Android devices with a minimum camera resolution of five megapixels to clearly capture the dog's skin and make a precise prediction. In this study, the Open Source Computer Vision (OpenCV) was used to detect and analyze captured image as a localized skin lesion, in which it has the ability to stitch the image together in order to produce a high-resolution image of the entire scene and to clearly find a similar data image from the images in the database. The artificial Neural Network (ANN) model was also utilized in the application for the training and testing of the dog skin disease dataset where the captured images can be detected and analyzed by using OpenCV. Captured images are compared to the sample images in the database and is used for image classification. Further, the application has the ability to analyze the captured image and provide the details when determined.
\end{abstract}

Key words: Artificial neural network, dog skin disease, image processing, mobile application, OpenCV

\section{INTRODUCTION}

Dogs are undeniably considered, as always, as man's best friend anywhere in the world, especially to the people who are truly pet lovers. Dogs understand what their master is feeling and are very sensitive too. These are just a few of the reasons why most of the people prefer to have a dog as their companion rather than any other pets they can have. No pet owners would want their pets to be ill, but it is an undeniable fact that these lovable creatures are likely to have a different kind of diseases, particularly with their skin, since it is the largest and most reachable organ of the body.

Comparable to humans; unfortunately, it is very common for dogs to have skin disorders. According to a local veterinarian, there are two types of skin disorders in dogs: the curable and the incurable kind of skin diseases. The curable ones vary from acute to self-limiting problems, whereas the incurable one is considered as a chronic (long-lasting) problem requiring lifetime treatment. Additionally, the incurable ones are usually hereditary and developmental skin disorders of dogs.

Despite the advent of technology, there is still limited mobile-based application intended for dogs that detects possible localized skin diseases and provides details of the detected skin disease informatively. Further, some local pet owners and guardians have limited knowledge as to what their pets might be experiencing. According to some interviewed local dog owners, dogs that have contracted skin diseases are just taken for granted and the changes that were observed as to dog's skin conditions are neglected because of the lack of time to visit a veterinarian that often results to a more serious skin problem that leads to other infections.

Therefore, this study aims to create a mobile application in the Android platform envisioned for the use of dog owners and provide them assistance in terms of medical findings in relation to the skin problem of their canines specifically with ringworm and yeast infections which are fungi related ailments. With this, a mobile application is designed to help dog owners identify possible skin diseases with the use of an Android-powered phone's rear camera by scanning the skin lesion of the dog. The application detects and analyzes images of captured localized skin lesions using the Open-source Computer Vision library and determines the skin disease and its respective description using the trained ANN model.

\section{RELATED SYSTEMS}

\subsection{Skin Infection Detection in Android using Image Processing}

The application can detect skin infection for the users to become more aware of their skin health status. The infected skin must be captured in order for the system to detect and scan the captured image using the OpenCV image processing 
feature. The system then posts the signs and symptoms, diagnosis, and treatment of the skin infected area [1].

\subsection{Segmentation and Classification of Skin Lesions for Disease Diagnosis}

Five classes of different skin infections are selected in this study, namely: Seborrheic keratosis, melanoma, bullae, squamous cell, and shingles. To remove the unwanted noise and hairs, the skin images are filtered, and to extract the lesion areas, the segmentation process is carried out. An application of pixel-based image segmentation was also made in the study where extracted color and texture features represent the lesion areas [2].

\subsection{Design and Development of Online Dog Diseases Diagnosing System}

The study focused on the dog's common diseases whose objective is to develop an expert system that can diagnose dog's diseases such as parvo, distemper, glaucoma, jaundice, colitis, kennel cough, virus, and leptospirosis in the near future. The study ensures that the abovementioned diseases are detected at an early stage. Further, the system assists dog owners in detecting diseases and provides suggestions for the treatment that generates a result and advice too [3].

\subsection{Skin Cancer Detection Using Artificial Neural Networking}

The aim of the study is to provide an automated application that assesses a patient's risk of having melanoma using the images of the skin lesions captured by the digital camera [4]. In the study, the good characteristics of neural networks were highlighted in relation to skin cancer image processing. The ANN has been used in cancer detection to deal with the matters that cannot be solved by other classification methods or by the traditional image processing.

\subsection{M-Health Skin: Disease Analysis System Using Smartphone's Camera}

The study proposed a smartphone-based application that allows people to examine the skin for skin diseases. M-Health solution is a smartphone-based skin health analyzer that uses mobile neural networks to distinguish normal skin images and abnormal skin images. To scan skin images, an intelligent learning algorithm, and a cell phone's camera was used [5].

\subsection{Automating skin disease diagnosis using image classification}

Skin cancer incidence is rapidly increasing, especially in Caucasian population countries that motivate this research. The study aims to minimize the dependency on the opinions of doctors by making use of a feature based on texture analysis and classify the lesion by utilizing the artificial neural network as a classification technique [6].

\section{METHODOLOGY}

\subsection{Data Gathering}

In the gathering of data, most of the information collected was from the interviewed staff and veterinary doctors in Davao City, Philippines. The dataset used in the study is from the dataset repository of local veterinary clinics in the locality.

\subsection{Development Tools}

\subsubsection{Open-source Computer Vision Library (OpenCV)}

This tool was instrumental in the study since it is a library of programming functions with more than 2500 algorithms that are optimized. It is also commonly used for mobile application development that uses image processing.

\subsubsection{Android Studio}

The Android Studio was instrumental in the study since it is the official integrated development environment (IDE) used for developing Android platform applications. IDE has developer tools and a powerful code editor. In addition, it offers more features that enhance the productivity and development of an android application.

\subsubsection{Android NDK}

The tool is instrumental so that the Android Studio library will be written in Java/C++.

\subsubsection{Android SDK}

The tool is used in developing the application on the Android platform.

\subsubsection{Programming Language: JAVA}

The Java programming language is used for the development of the android application. This open-source programming language is considered to be one of the most diverse programming languages to be used for the development of any system.

\subsubsection{Neuroph}

The Neuroph framework is an object-oriented neural network which is written in Java and can be used to train the neural network. It also provides a GUI tool as well as the Java class library for preparing and creating the neural application and neural networks training.

\subsubsection{SQLite}

The SQLite is used as a storage of collected input and output data. 


\subsection{Conceptual Framework}

The methodology for development used in this study is the Agile Development and are in forms of the sprint to wit: requirement analysis, planning, design, development, maintenance, and implementation.

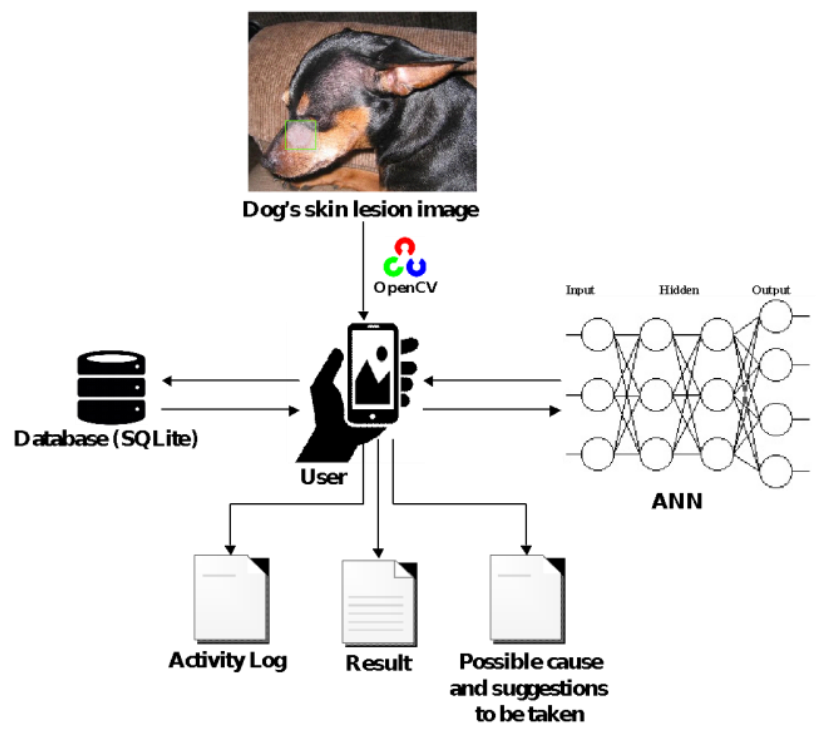

Figure 1: Conceptual framework of the system

Figure 1 illustrates the overall process of the mobile application. The user takes an image of a skin lesion from a dog using an Android phone with a camera of at least five megapixels to generate a quality image. The captured image is sent to OpenCV. For the skin lesion to be detected and to be analyzed, the captured image undergoes a preprocessing method to clear the unwanted parts of the dog from the captured image leaving only the affected skin of the dog. The feature extraction method is done using OpenCV, where all the necessary features are acquired and converted into values which can be fed into the neural network where the identification of the skin disease occurs. The identified skin disease of the captured image from the dog is generated; then an output displays on the phone screen of the user associated with the possible causes of such skin disease acquired by the dog and some tips and suggestions to be taken by the user for the wellness of the infected dog.
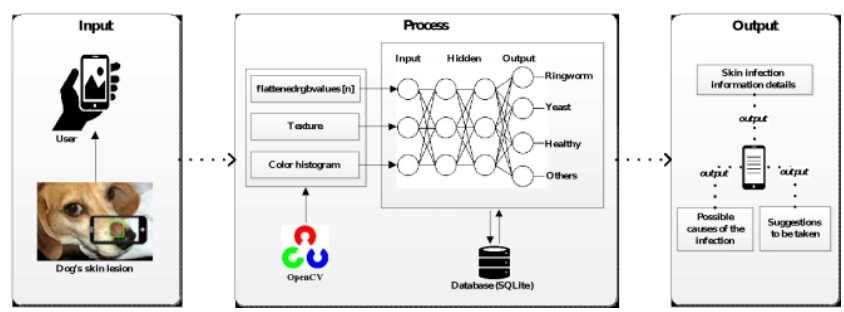

Figure 2: The input, process, and output of the application

Figure 2 illustrates the entire flow of the mobile application, together with the tools integrated to build and develop an Android-based application. The flow begins when the user captures the affected spot of the canine's skin by the camera of an Android phone wherein detecting of the skin is done by using OpenCV. A green tick mark or rectangular figure is drawn around the detected skin lesion where the affected skin region is cropped and forwarded for image preprocessing, segmentation, and extraction methods.

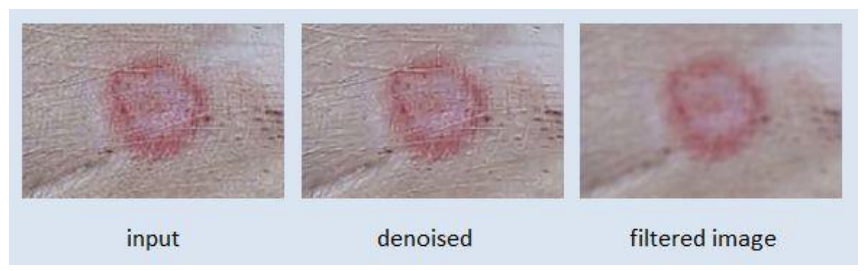

Figure 3: Sample image preprocessing

Figure 3 shows the first step in the skin lesion image analysis, which is the pre-processing. Filtering is done to remove noise and Gaussian operation takes place to smoothen and enhance the image.

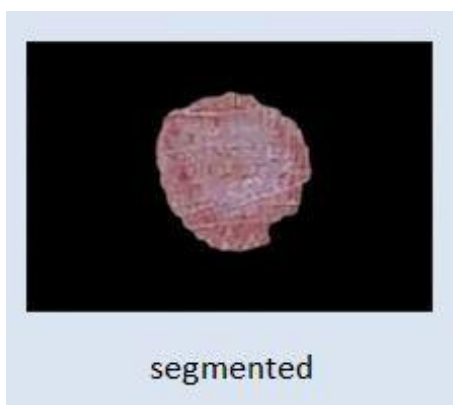

Figure 4: Segmentation

To further enhance the filtered image, the segmentation process takes place with the sample image, as shown in Figure 4. The process isolates and/or separates the object in the image and assigns a label to every pixel. The next process is the feature extraction where skin lesion images are characterized by the features that have been used. To represent the lesion areas, the texture, color, and color histogram features are used.

In order to get the color that exists in a skin lesion, the three two-dimensional arrays are used to represent an image in RGB values for one for each of the color components, where element corresponds to one pixel such that location $[24,16]$ has color RGB [35, 88, 183] with redValues $(24)(16)=35$; greenValues $(24)(16)=88$; and blueValues $(24)(16)=183$.

Each of the arrays has the dimension represented with [imageHeight][imageWidth]. The three arrays are merged into a single one-dimensional array, so it contains all red, green, and blue values to create a flattened RGB values array to be used as an input for the neural network.

To get the texture present in a lesion, the gray level co-occurrence matrix can be employed [2]. GLCM is an $\mathrm{M} \times \mathrm{M}$ square matrix, where $\mathrm{M}$ is the different gray levels in the image. Texture features (co-occurrence) in the image were extracted in two steps. (1) Using a GLCM, the spatial co-occurrence of separated pixel by an exact distance and angle is developed. (2) The gray level co-occurrence matrix is used to calculate the set of the scalar quantity, which distinguishes the different aspects of the original texture. In 
order to represent the color histogram features, the general understanding regarding the brightness, contrast, and intensity distribution in the image must be examined.

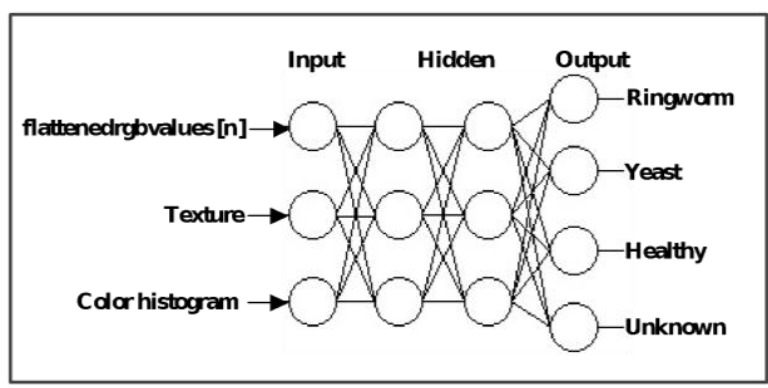

Figure 5: Artificial Neural Network

Figure 5 shows the graphical representation of the neural network. This computational model of an algorithm, which is based on the functions and structure of a biological neural network, is used in this study. Neural networks are utilized for image recognition. The Neuroph has an integrated backing system for image recognition with a dedicated wizard for training image recognition neural networks.

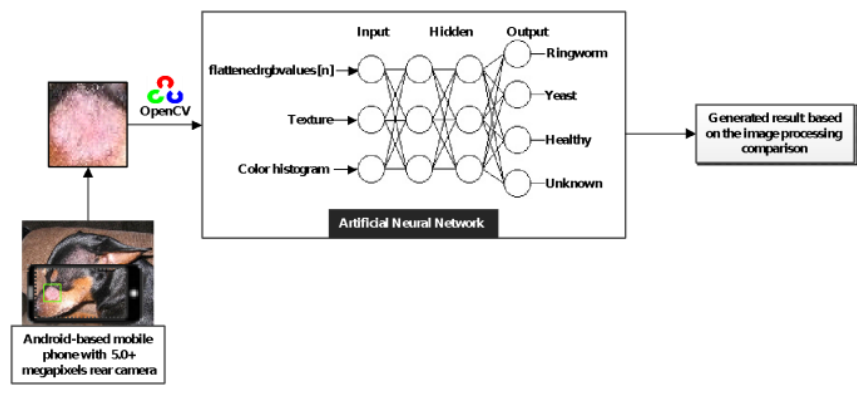

Figure 6: Process of determining the skin disease

Figure 6 shows the illustration of the process in determining the canine skin disease, whether it is ringworm or a yeast infection. After the captured image went through the image processing phase, features of such preprocessed images are now extracted and converted. The right value of each feature, such as the color, texture, and color histogram from the processed image is fed into the neural network as an input to be compared to the network outputs, which are learned by the neural network.

The network has four outputs which have class labels, namely (1.) Ringworm, (2.) Yeast, (3.) Healthy and (4.) Others that corresponds to the output neurons. The recognized image is matched to the neuron with the highest output. The Healthy class output concerns with the image that has no sign of any skin diseases while the 'Others' class output pertains to the skin diseases other than ringworm and yeast infections which are not covered in the study.

After the process of analyzing the image, the application then shows the result whether the scanned image of the skin lesion is ringworm or a yeast infection. The application shows a brief description of the information for its output and the causes of the infection. The application also displays some tips and suggestions the dog owner must adhere. In short, the application serves as a supplemental guide to dog owners on how to deal with the identified skin problem.

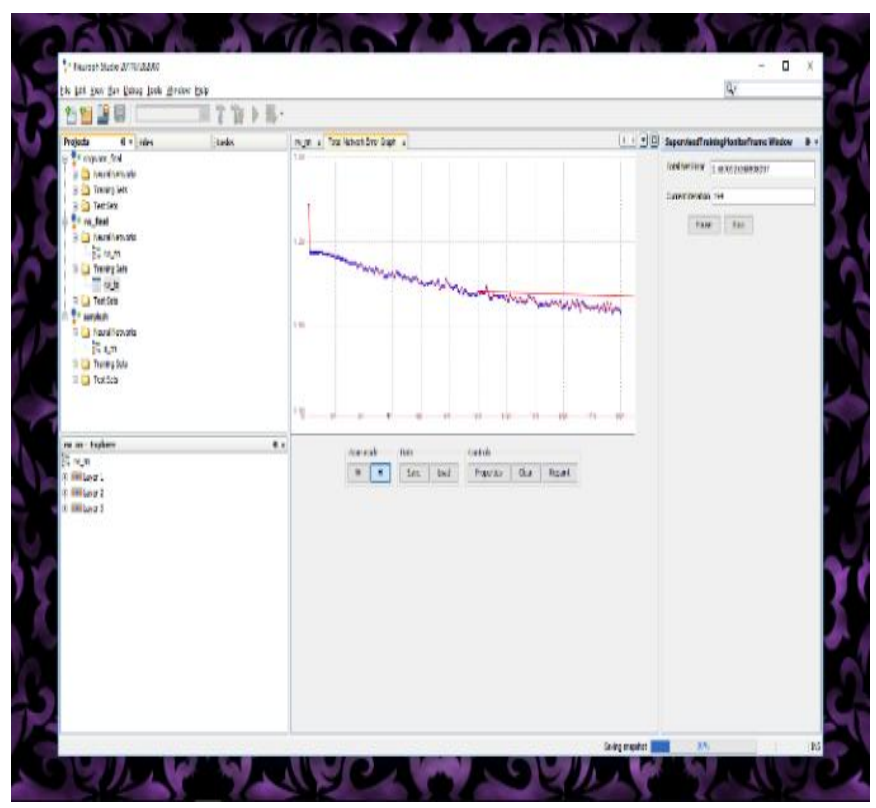

Figure 7: Dataset training

The dataset used in this study are the 200 ringworm and 200 yeast infection images that were trained and tested in Neuroph Studio as shown in Figure 7. Captured images are compared to the trained dataset where an image that is positive for yeast and ringworm infection meets the value set to 0.01 limit; otherwise, the image is not infected and is placed in junked files.

\section{RESULTS AND DISCUSSION}

\subsection{Prototype}

This study creates a mobile application that detects localized skin disease for dogs with the help of image processing via the artificial neural network through the Neuroph framework. Figures 8-22 shows the graphical representation of the functionalities of the system.
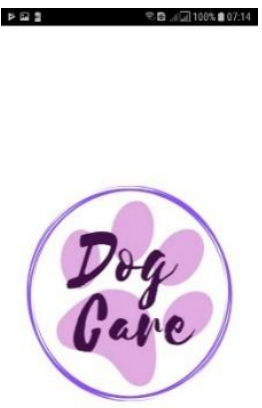

Figure 8: Splash screen

Figure 8 shows the splash screen of the application upon loading on the mobile phone. 
Figure 9 shows the main menu page of the application where test, result, clinic, and information modules are located and accessed.

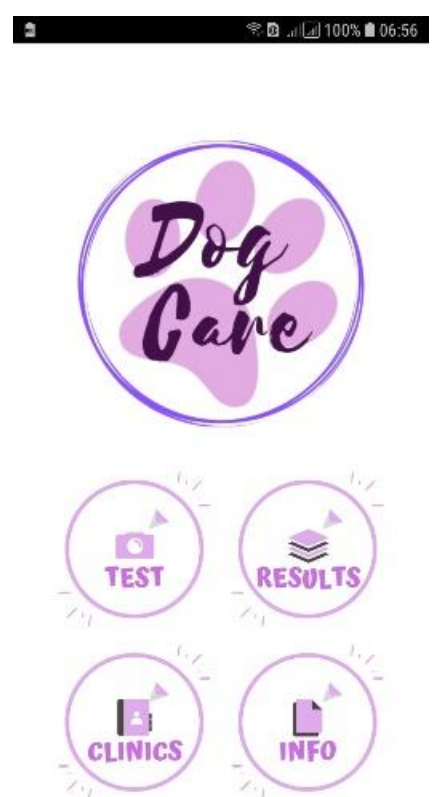

Figure 9: App main menu

Figure 10 shows the page where users are asked for the source of the image to be tested.

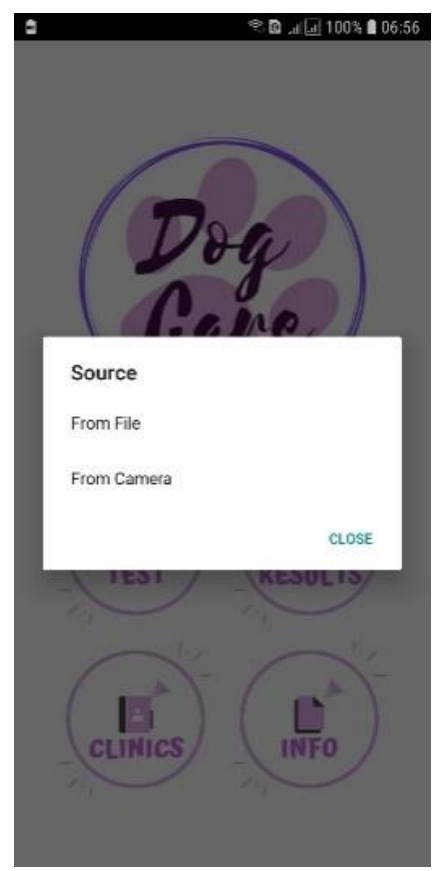

Figure 10: Image source for test option

Figure 11 shows the result of the image detection that has been captured and tested. The type of skin disease, its description, and symptoms are shown in the app.

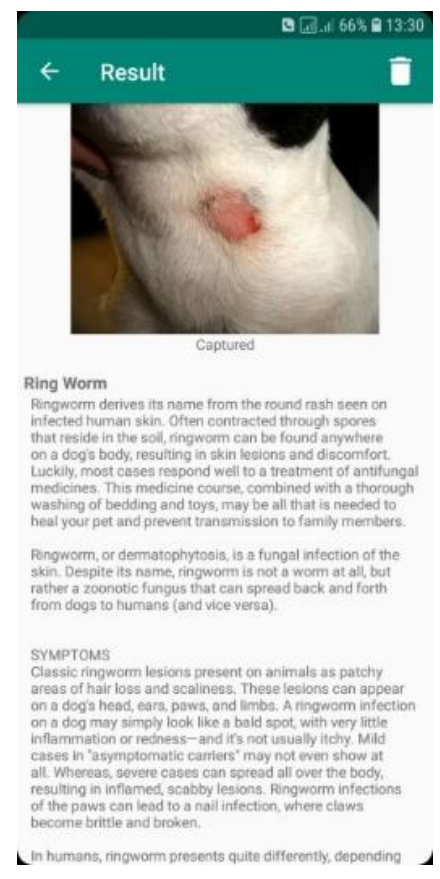

Figure 11: Test result - ringworm detection

Figure 12 shows the result of the tested image where the type of skin disease, corresponding percentage accuracy of the classification, and recommended remedy from the tested image is shown in the app report.

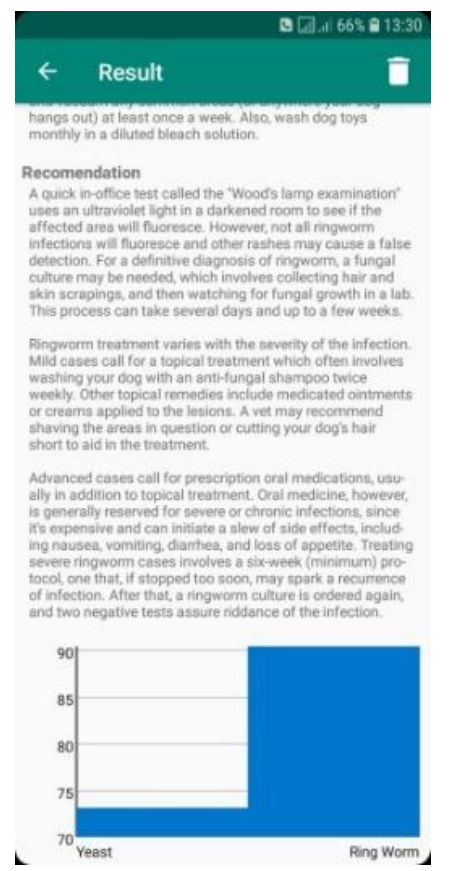

Figure 12: Test result - ringworm detection

Figure 13 shows the list of skin diseases that can be detected by the application. 


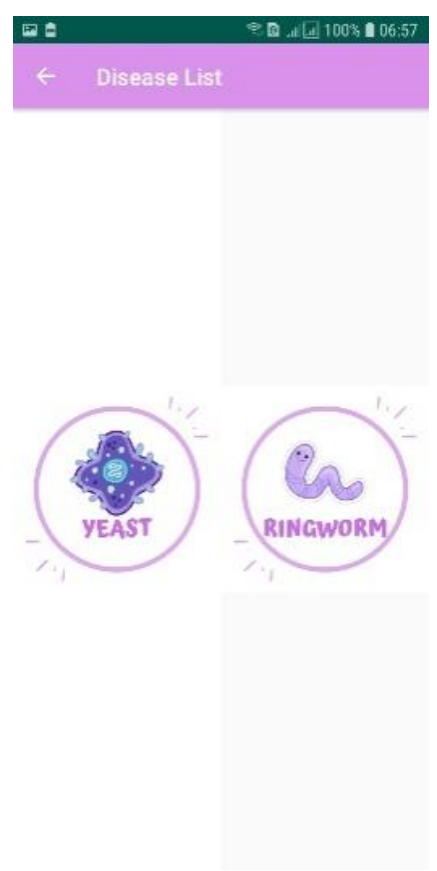

Figure 13: Disease list

Figure 14 shows the information and symptoms of the disease found in the disease list module.

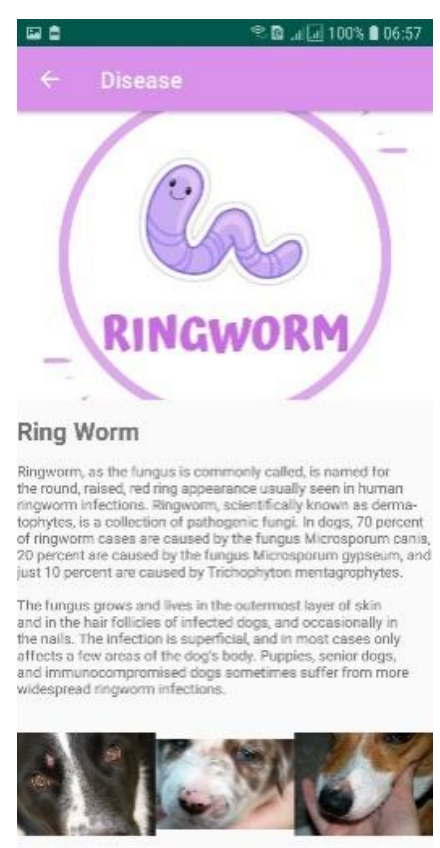

Figure 14: Disease information

Figure 15 shows the content of the clinic module where the necessary details are listed with respect to the veterinary doctors and clinics found in Davao City, Philippines. Personal information such as the doctor's name and contact number are presented in the system for easy consultation and appointment undertakings.

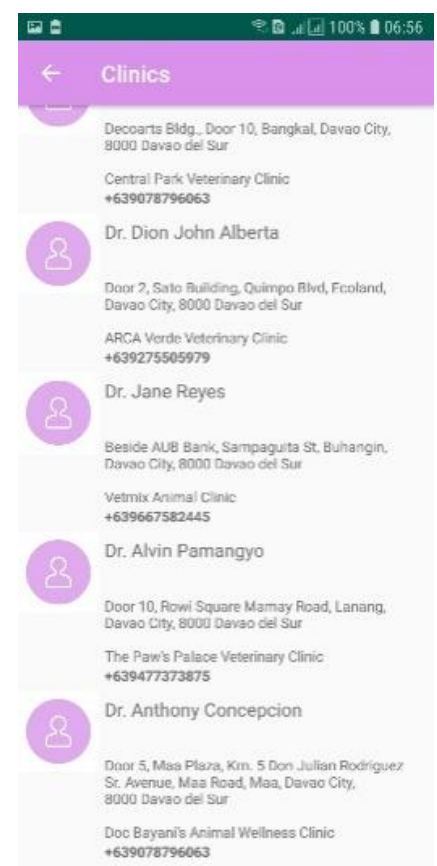

Figure 15: Clinics module

\subsection{Compatibility Assessment}

In this study, several tests that determine the usability of the developed system were conducted to wit: android version test, camera resolution test, RAM test, disease capture distance test, and lighting test. Tables 1-6 shows the result of the conducted compatibility assessment.

Table 1: Android version testing

\begin{tabular}{|l|c|}
\hline \multicolumn{1}{|c|}{ Android OS Version } & Remarks \\
\hline Android 4.4.2 Kitkat & Passed \\
\hline Android 5.1.1 Lollipop & Passed \\
\hline Android 6.0.1 Marshmallow & Passed \\
\hline Android 7.1.1 Nougat & Passed \\
\hline Android 8.1.0 Oreo & Passed \\
\hline
\end{tabular}

Table 2: Camera resolution testing

\begin{tabular}{|l|c|}
\hline \multicolumn{1}{|c|}{ Camera Resolution } & Remarks \\
\hline 5-Megapixel & Passed \\
\hline 8-Megapixel & Passed \\
\hline 13-Megapixel & Passed \\
\hline 16-Megapixel & Passed \\
\hline
\end{tabular}

Table 3: RAM testing

\begin{tabular}{|l|c|}
\hline \multicolumn{1}{|c|}{ RAM Capacity } & Performance and Remarks \\
\hline 512MB RAM & Working \\
\hline 1GB RAM & Working \\
\hline 2GB RAM & Working \\
\hline 3GM RAM & Working \\
\hline
\end{tabular}

Table 4: Capture distance testing - ringworm

\begin{tabular}{|c|c|}
\hline Distance & Remarks \\
\hline 0.25 Feet & Passed \\
\hline 0.5 Feet & Passed \\
\hline 1 Feet & Passed \\
\hline 2 Feet & Passed \\
\hline 3 Feet & Failed \\
\hline
\end{tabular}


Table 5: Capture distance testing - yeast

\begin{tabular}{|c|c|}
\hline Distance & Remarks \\
\hline 0.25 Feet & Passed \\
\hline 0.5 Feet & Passed \\
\hline Feet & Passed \\
\hline 2 Feet & Failed \\
\hline 3 Feet & Failed \\
\hline
\end{tabular}

Table 6: Light test

\begin{tabular}{|l|c|}
\hline \multicolumn{1}{|c|}{ Types of Lighting Distance } & Remarks \\
\hline Natural Light & Passed \\
\hline Controlled Light & Passed \\
\hline Hard Light & Failed \\
\hline Soft Light & Passed \\
\hline
\end{tabular}

The app was tested on five different versions of Android phones, along with various camera megapixels and memory capacity. The result shows that the app is recommended to be installed on phones with a minimum of 5 megapixels camera and a RAM capacity higher and not less than $512 \mathrm{mb}$. Further, in capturing the skin lesion, the suggested distance from the dog's spot must not be more than 1 feet from the infected part of the dog. Furthermore, it is advised that the user should capture an image in a bright surrounding.

\subsection{Usability Assessment}

Tables 7-8 shows the result of the usability test conducted with regards to the functionalities and modules of the application.

Table 7: Functionality testing

\begin{tabular}{|l|l|}
\hline \multicolumn{1}{|c|}{ Objective } & Result \\
\hline Scan and capture image & Passed \\
\hline Test the captured image & Passed \\
\hline Display the test result & Passed \\
\hline Determine the type of skin disease & Passed \\
\hline Give info about the skin disease & Passed \\
\hline Display and contact info of Veterinarian & Passed \\
\hline
\end{tabular}

Table 8: Module testing

\begin{tabular}{|l|c|c|c|c|c|}
\hline & \multicolumn{5}{|c|}{ Module Testing in Different Devices } \\
\hline \multirow{3}{*}{$\begin{array}{l}\text { Functions/ } \\
\text { Modules }\end{array}$} & \multicolumn{5}{|c|}{ Android Devices } \\
\cline { 2 - 6 } & Samsung & Lenovo & Samsung & Starmob & -ile \\
& Galaxy & S9 & Galaxy & VLAY & Y69 \\
& A6 2018 & -A90 & A5 2016 & LiTE & \\
\hline Splash & Passed & Passed & Passed & Passed & Passed \\
\hline Main Menu & Passed & Passed & Passed & Passed & Passed \\
\hline Test Option & Passed & Passed & Passed & Passed & Passed \\
\hline Result & Passed & Passed & Passed & Passed & Passed \\
\hline Disease List & Passed & Passed & Passed & Passed & Passed \\
\hline Veterinaries & Passed & Passed & Passed & Passed & Passed \\
\hline
\end{tabular}

Results show that the application works properly in accordance with the set objectives and functionalities of the proposed system.

\subsection{Prediction Validation}

A cross-checking process was done with the presence of a local veterinarian where he was presented with five pictures for ringworms and five pictures for yeast infections for personal validation. Result revealed that in Ringworm test, Test 3 of Picture 1, Test 4 of Picture 2 and Test 2 of Picture 4 failed to the validation prediction of the local veterinarian. On Yeast Test, Test 2 of Picture 1 and Test 4 of Picture 4 failed in the validation process, as shown in Table 9. Out of the 25 tests taken from each for the Ringworm and Yeast Test, the application obtained a $97 \%$ and $98 \%$ prediction accuracy for the correctly classified images for ringworm and yeast test, respectively. This proved that the algorithm detects skin disease correctly.

Table 9: Indexed validation result

\begin{tabular}{|c|c|c|c|c|c|}
\hline Ringworm Test & Test1 & Test 2 & Test 3 & Test 4 & Test 5 \\
\hline Picture 1 & $\checkmark$ & $\checkmark$ & $X$ & $\checkmark$ & $\checkmark$ \\
\hline Picture 2 & $\checkmark$ & $\checkmark$ & $\checkmark$ & $X$ & $\checkmark$ \\
\hline Picture 3 & $\checkmark$ & $\checkmark$ & $\checkmark$ & $\checkmark$ & $\checkmark$ \\
\hline Picture 4 & $\checkmark$ & $X$ & $\checkmark$ & $\checkmark$ & $\checkmark$ \\
\hline Picture 5 & $\checkmark$ & $\checkmark$ & $\checkmark$ & $\checkmark$ & $\checkmark$ \\
\hline Yeast Test & Test1 & Test 2 & Test 3 & Test 4 & Test 5 \\
\hline Picture 1 & $\checkmark$ & $X$ & $\checkmark$ & $\checkmark$ & $\checkmark$ \\
\hline Picture 2 & $\checkmark$ & $\checkmark$ & $\checkmark$ & $\checkmark$ & $\checkmark$ \\
\hline Picture 3 & $\checkmark$ & $\checkmark$ & $\checkmark$ & $\checkmark$ & $\checkmark$ \\
\hline Picture 4 & $\checkmark$ & $\checkmark$ & $\checkmark$ & $X$ & $\checkmark$ \\
\hline Picture 5 & $\checkmark$ & $\checkmark$ & $\checkmark$ & $\checkmark$ & $\checkmark$ \\
\hline
\end{tabular}

\section{CONCLUSION}

The objectives discussed in this study have been achieved as results show that the developed application can capture a localized skin lesion using the phone's rear camera. Further, the app is used to detect and analyze dog skin lesion using the OpenCV library and determining skin diseases are made possible with the use of an artificial neural network. It is concluded that the diagnosis and treatment of dog's skin diseases are made possible at home with the help of the proposed application.

\section{REFERENCES}

[1] A. Carlon, J. Estrera, and B. Galope, "Skin Infection Detection in Android using Image Processing," 2014.

[2] R. Sumithra, M. Suhil, and D. S. Guru, "Segmentation and classification of skin lesions for disease diagnosis," Procedia Comput. Sci., vol. 45, pp. 76-85, 2015. https://doi.org/10.1016/j.procs.2015.03.090

[3] M. Y. Munirah, S. Suriawati, and P. P. Teresa, "Design and Development of Online Dog Diseases Diagnosing System," Int. J. Inf. Educ. Technol., vol. 6, no. 11, pp. 913-916, 2016. https://doi.org/10.7763/IJIET.2016.V6.816

[4] A. Antony, A. Ramesh, A. Sojan, B. Mathews, and M. T. A. Varghese, "Skin cancer detection using artificial neural networking," Skin (Los. Angeles)., vol. 4, no. 4, 2016.

[5] A. Bourouis, A. Zerdazi, M. Feham, and A. Bouchachia, "M-health: Skin disease analysis system using smartphone's camera," Procedia Comput. Sci., vol. 19, pp. 1116-1120, 2013.

https://doi.org/10.1016/j.procs.2013.06.157

[6] D. A. Okuboyejo, O. O. Olugbara, and S. A. Odunaike, "Automating skin disease diagnosis using image classification," in Proceedings of the World Congress on Engineering and Computer Science, 2013, vol. 2. 